\title{
NON-INVASIVE MEASUREMENT OF THREE DIMENSIONAL JOINT ANGLES: DEVELOPMENT AND EVALUATION OF A COMPUTER-AIDED SYSTEM FOR MEASURING WORKING POSTURES
}

\author{
W. Monroe Keyserling and Peter M. Budnick \\ Center for Ergonomics, Department of Industrial and Operations Engineering, The University of Michigan, \\ 1205 Beal Avenue, Ann Arbor, MI 48109-2117 (U.S.A.)
}

(Received August 17, 1986; accepted in revised form October 24, 1986)

\section{ABSTRACT}

A new system was developed to analyze work posture in order to measure the three-dimensional body angles required as inputs to a biomechanical strength prediction model. This system was non-invasive and required no interference with work activities. Videotape was used at the job site to establish a permanent record of postural activity and a computer-aided digitization system was used to measure body angles in the laboratory.

An experiment using five subjects and ten work postures was performed to investigate the nature and significance of measurement errors when using the new system. The results of this experiment revealed that, in general, subjects were able to distinguish different work postures; and that inter-subject bias was not significant. Measurement error was small for most joint angles and could be reduced further by enhancements to the system hardware. The system was found to be a useful tool for determining threedimensional body angles when direct measurements are infeasible.

\section{INTRODUCTION}

Despite recent advances in the development of mechanized materials handling systems for industry (e.g., hoists, lift trucks, conveyors, robots, etc.), it is estimated that approximately one-third of all workers in the United States must regularly exert significant strength to lift, push, or pull objects as part of their jobs. Overexertion stresses associated with these activities frequently result in lost-time injuries such as sprains and strains of musculoskeletal tissues in the back, shoulders, and other joints. The cost of these injuries is not trivial; a recent analysis of Workers' Compensa- tion records by the National Institute of Occupational Safety and Health (NIOSH) revealed that overexertion injuries accounted for approximately one-fourth of all occupational injury claims filed in the United States. Manual materials handling activities such as lifting, pushing, or pulling were cited as contributing factors in about 85 percent of these claims (NIOSH, 1981).

Recent studies have concluded that several job and task factors contribute to the risk of overexertion injuries during manual materials handling, including:

(a) The magnitude and direction of the resultant force when lifting or moving an object 
(Chaffin and Park., 1973; Snook, 1978; Ayoub et al., 1978; NIOSH, 1981). For example, in a controlled carrying task, the magnitude of the resultant force is equal to the object's weight while the direction of the resultant force is down.

(b) The frequency and duration of the materials handling activities (Snook, 1978; NIOSH, 1981).

(c) The body posture maintained while handling the object (Chaffin and Park, 1973; Snook, 1978; Chaffin et al., 1977a; Ayoub et al., 1978; NIOSH, 1981). Body posture is frequently determined or affected by job variables such as workstation layout and the size/shape of the handled object.

Of the above factors, the resultant force and frequency/duration are relatively easy to measure and describe. Force can usually be measured with a simple spring scale and described as a vector. Frequency and duration of materials handling activities can be determined from production records and/or direct observation.

Posture, however, can be very difficult to measure and describe due to the numerous articulations of the human body and multiple degrees of freedom at certain joints. For example, a relatively simple two-dimensional biomechanical model used to evaluate the static strength requirements of sagittal-plane lifting, pushing, and pulling tasks requires the measurement of angles at five joints: the elbow, shoulder, hip, knee and ankle (Chaffin, 1969). In general, these measurements are relatively easy to make and describe because limb motions and joint angles are constrained to the sagittal plane. See Fig. 1a for an illustration of these angles. A three-dimensional extension of this biomechanical model (i.e., one that is no longer restricted to sagittal plane activities) requires the determination of 15 angles in order to evaluate the strength demands of a task (Garg and Chaffin, 1975). In this model, certain joints (e.g., the L5/S1 spinal joint and the shoulders) have multi-directional axes of motion. Furthermore, joint angles may be defined in arbitrary planes depending upon the relative position of adjacent limbs. See Fig. $1 b$ and Table 1 for a description of these angles.

In previous applications of the three-dimensional model, body angles have been directly measured on the worker (using hand-held goniometers), estimated from digitized positional data for adjacent joints (e.g., photographic and/or Selspot methods), or estimated using an algorithm driven by a general description of posture (e.g., stand, stoop, squat, etc.) and the Cartesian coordinates of the worker's hands (Chaffin et al., 1977b). The direct measurement technique proves impractical in most work situations because it requires the worker to "freeze" while the angle measurements are taken, a time consuming process that may interfere with body movement dynamics and/or productivity. The digitization techniques permit natural movements and can be used to collect data in real time. These methods generally work well in the laboratory, but prove impractical in many field situations due to hardware reliability problems and calibration requirements. The Cartesian

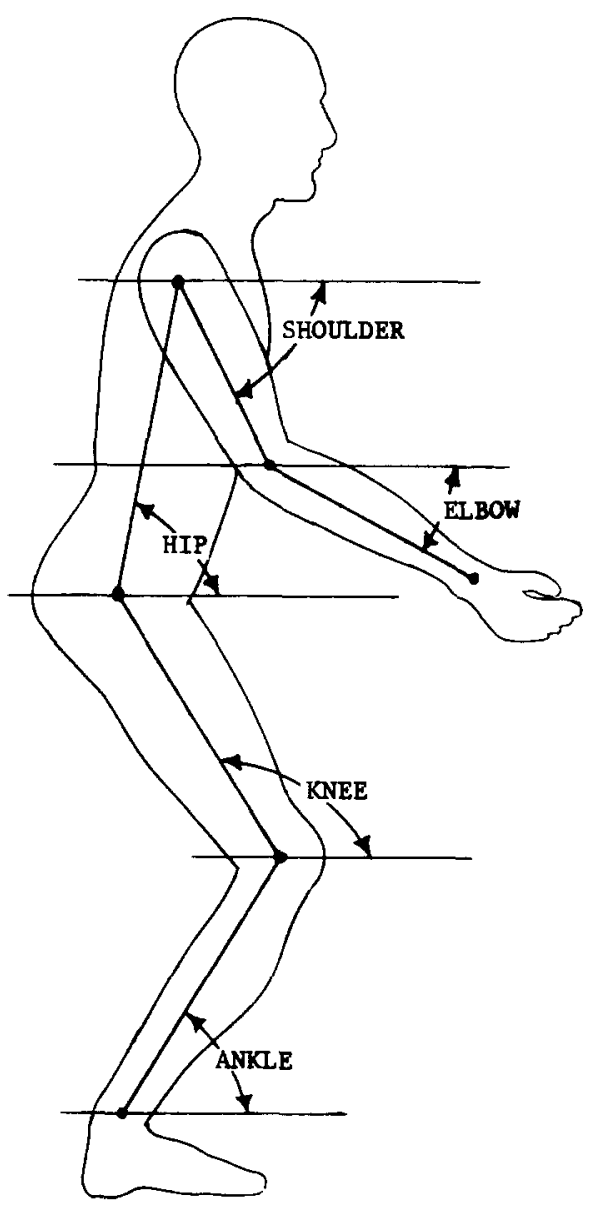

Fig. 1(a). The five angles required for the 2-dimensional sagittal plane biomechanical strength model (adapted from Chaffin, 1969). 


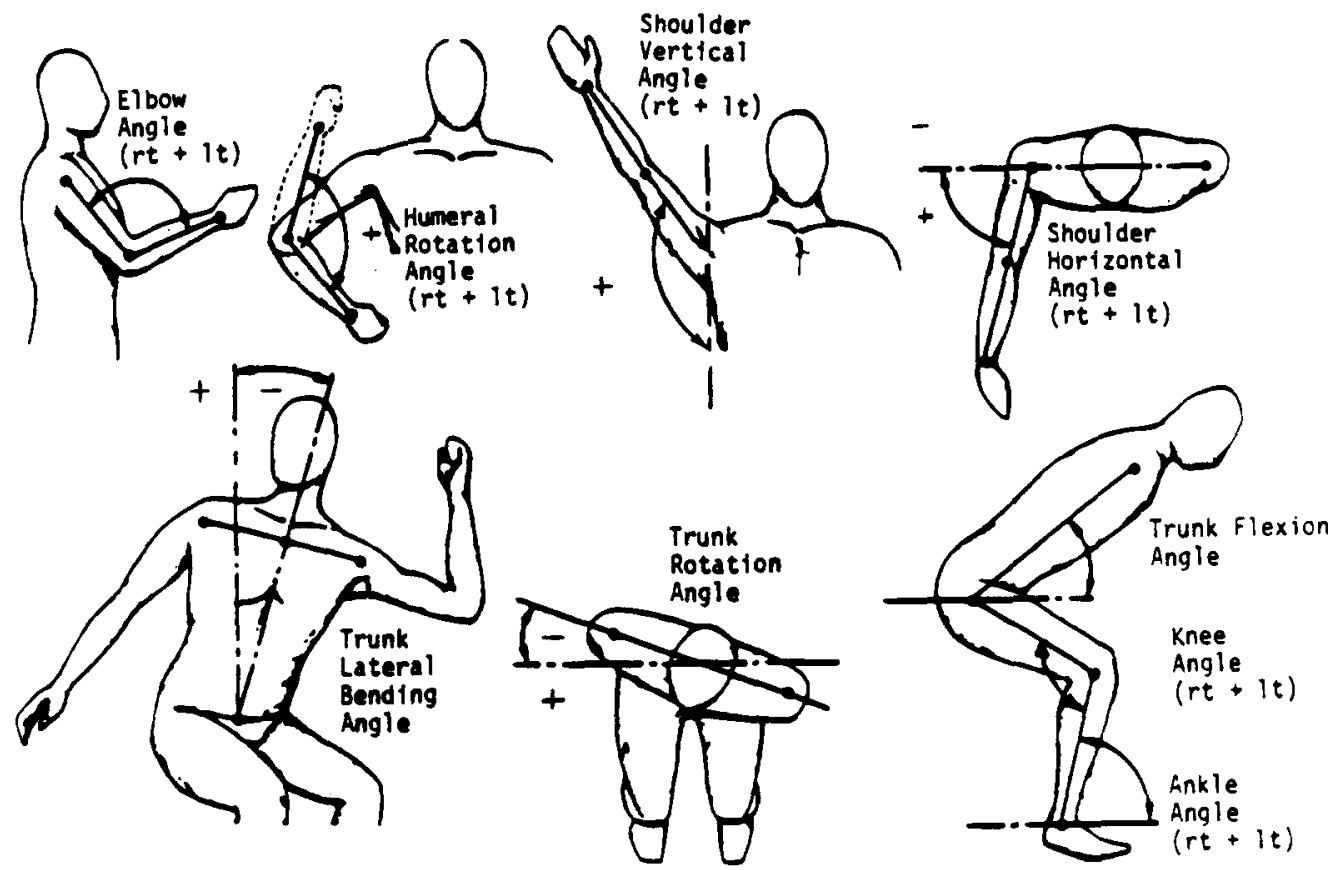

Fig. 1(b). The 15 angles required for the 3-dimensional biomechanical model (adapted from Garg and Chaffin, 1975).

TABLE 1

Descriptions of the 15 angles required for the 3-dimensional biomechanical model

\begin{tabular}{|c|c|c|}
\hline Angle & Description & Sign \\
\hline Right and left elbow & $\begin{array}{l}\text { The included angle between the upper arm } \\
\text { and lower arm }\end{array}$ & Always positive \\
\hline $\begin{array}{l}\text { Right and left } \\
\text { shoulder vertical }\end{array}$ & $\begin{array}{l}\text { The angle between the upper arm } \\
\text { and the trunk }\end{array}$ & Always positive \\
\hline $\begin{array}{l}\text { Right and left } \\
\text { shoulder horizontal }\end{array}$ & $\begin{array}{l}\text { The angle between the upper arm and the } \\
\text { frontal plane when viewed from above }\end{array}$ & $\begin{array}{l}\text { Positive when upper arm } \\
\text { is anterior to the frontal } \\
\text { plane, negative when posterior }\end{array}$ \\
\hline $\begin{array}{l}\text { Right and left } \\
\text { humeral rotation }\end{array}$ & $\begin{array}{l}\text { Axial rotation of the humerus, measured } \\
\text { from a predefined neutral position. } \\
\text { See Figure } 1 b \text {. }\end{array}$ & $\begin{array}{l}\text { Medial rotation is } \\
\text { positive, lateral } \\
\text { rotation is negative }\end{array}$ \\
\hline Trunk flexion & $\begin{array}{l}\text { The angle between the trunk and the } \\
\text { horizontal, measured in the sagittal plane }\end{array}$ & Always positive \\
\hline Trunk lateral bending & $\begin{array}{l}\text { The angle between the trunk and the vertical, } \\
\text { measured in the frontal plane }\end{array}$ & $\begin{array}{l}\text { Positive when bent to right, } \\
\text { negative when bent to left }\end{array}$ \\
\hline Trunk rotation & $\begin{array}{l}\text { The angle of axial rotation, measured from } \\
\text { the sagittal plane }\end{array}$ & $\begin{array}{l}\text { Positive when right } \\
\text { shoulder is anterior to } \\
\text { sagittal plane, negative } \\
\text { when posterior }\end{array}$ \\
\hline Right and left knee & $\begin{array}{l}\text { The included angle between the upper leg and } \\
\text { lower leg }\end{array}$ & Always positive \\
\hline Right and left ankle & Angle of the lower leg above the horizontal & Always positive \\
\hline
\end{tabular}


coordinates approach is relatively quick and easy to use, but lacks in precision and is not well suited for tasks that involve unusual work postures.

The problems associated with measuring posture in a work setting are complicated by the fact that the position of the body can frequently change during manual materials handling activities. As a result, real-time posture measurement has been a challenge for ergonomists even when a small number of joints and body angles are being evaluated (Keyserling, 1986).

Several systems for recording posture have been specifically developed for the evaluation of occupational activities. Karhu et al. (1977) developed the Ovako Working Posture Analysis System (OWAS) to record posture during manual materials handling and other activities. To use this system, the analyst makes an instantaneous observation of posture and records a three digit code. The first digit describes the position of the trunk (four choices), the second digit describes the arms (three choices), and the third digit describes the legs (seven choices). Because the entire process of observing, coding, and recording requires only a few seconds, OWAS provides an efficient technique for documenting posture. The convenience of OWAS makes it impractical, however, for use in conjunction with the biomechanical models discussed above. Specifically, the standard posture categories used in the OWAS system are too broad to provide the level of precision required to describe the body angles which are used by the models.

Following the introduction of OWAS, Corlett et al. (1979) developed a system called "Posture Targeting". With this system, the analyst observes the worker and records the position of the head, trunk, upper arms, lower arms, upper legs, and lower legs by marking ten targets on a chart. These targets describe the angle of each body segment with respect to a standard reference position. For a static posture, a trained analyst can observe and record the required angles in about 30 seconds. (If the job requires frequent posture changes, a photograph can be used to freeze a posture for analysis.) Although the standard body angles measured with the Posture Targeting technique cannot be used directly by the biomechanical models discussed above, these angles can be transformed by computer to provide the necessary model inputs.
Other systems have been developed to measure the posture of specific joints and limbs during work activities. Armstrong et al. (1982) developed a cinemagraphic system and taxonomy for measuring and generating a detailed record of the posture of the upper extremities. Nordin (1982) developed a portable instrument for direct, realtime measurement of trunk movements in the sagittal plane. Persson and Kilbom (1983) developed a videotape-based system called VIRA to evaluate the posture of the neck and shoulders. A similar system was developed by Keyserling (1986) to evaluate the posture of the shoulders and trunk for standing assembly line work. While all of the above systems can be used to measure and evaluate specific types of postural stress, they do not measure all of the body angles necessary to describe posture as required for biomechanical strength models. To overcome this problem, a new method for collecting postural data has been developed and is described below.

\section{EQUIPMENT AND METHODS}

The following equipment and software were used to collect the data and compute the postural angles:

(a) A colour video camera and recorder, playback unit, and monitor. The camera and recorder must be sufficiently portable for use in the field. The playback unit must have the capability to display video "freeze frames" with high resolution and low distortion.

(b) A force measurement device (e.g., a spring scale or similar system) for measuring the magnitude of forces exerted by a worker during materials handling activities.

(c) A flexible three-dimensional human manikin for simulating the working postures during strenuous manual materials handling activities. In this study, a Loew-Cornell $36 \mathrm{~cm}$ (12 in), flexible, three dimensional, wood manikin was used. Scaling of body segments on the manikin was proportional to 50th percentile male anthropometry.

(d) A microcomputer-based digitization system for determining the Cartesian coordinates of joint locations on the manikin. A Micro Control Systems HIPI Space Tablet (a three dimensional digitizing system) was used in this study, along with a 
HIPI adaptor board for the IBM-PC, and HIPI Advanced Space Graphics software.

(e) Software for computing joint angles based on the coordinates of body joints measured with the HIPI "Space Tablet" system. A program called "POSTURE-3D" was developed by the investigators for this purpose (Univ. of Michigan, 1985).

(f) An IBM personal computer (or a compatible machine) equipped with two floppy disk drives and 256 kilobytes of memory.

A typical set-up of this equipment in a laboratory work station is illustrated in Fig. 2. In addition to the above standard equipment, it is sometimes desirable to take photographs or slides in order to create a permanent record of selected "freeze frames" from the videotape. Virtually any $35 \mathrm{~mm}$ SLR camera with manual focus and exposure controls is sufficient for this purpose.

A flowchart describing the use of the system is presented in Fig. 3. The portable video camera and recorder are used at the worksite to establish a permanent record of the operator performing the job. When making the tape, it is important that the camera angle be chosen so that all joints of interest can be clearly seen during playback. Several cycles of the job should be recorded because postures and tasks can vary from cycle to cycle. Any irregular activities that require significant strength demands (e.g., replenishing stock, changing tooling, etc.) should also be recorded. (On irregular jobs, such as maintenance work,

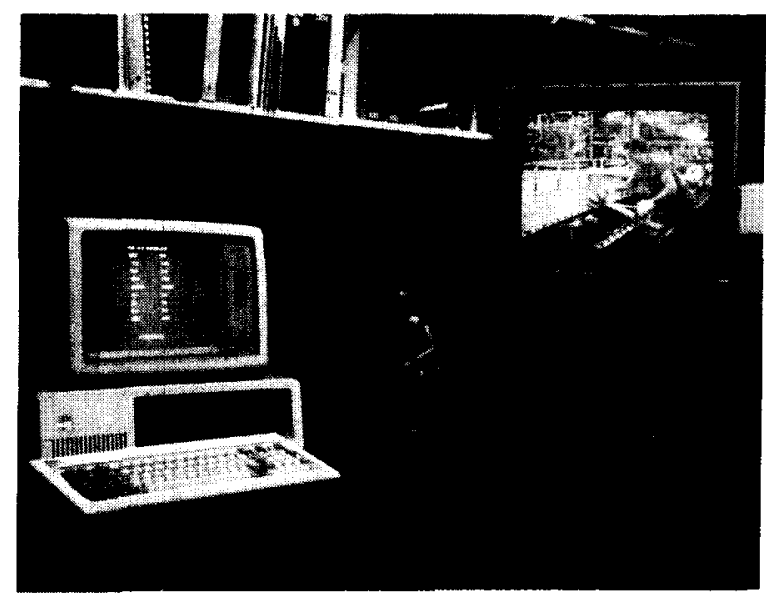

Fig. 2. Typical set-up of the laboratory equipment needed for the posture digitization method.

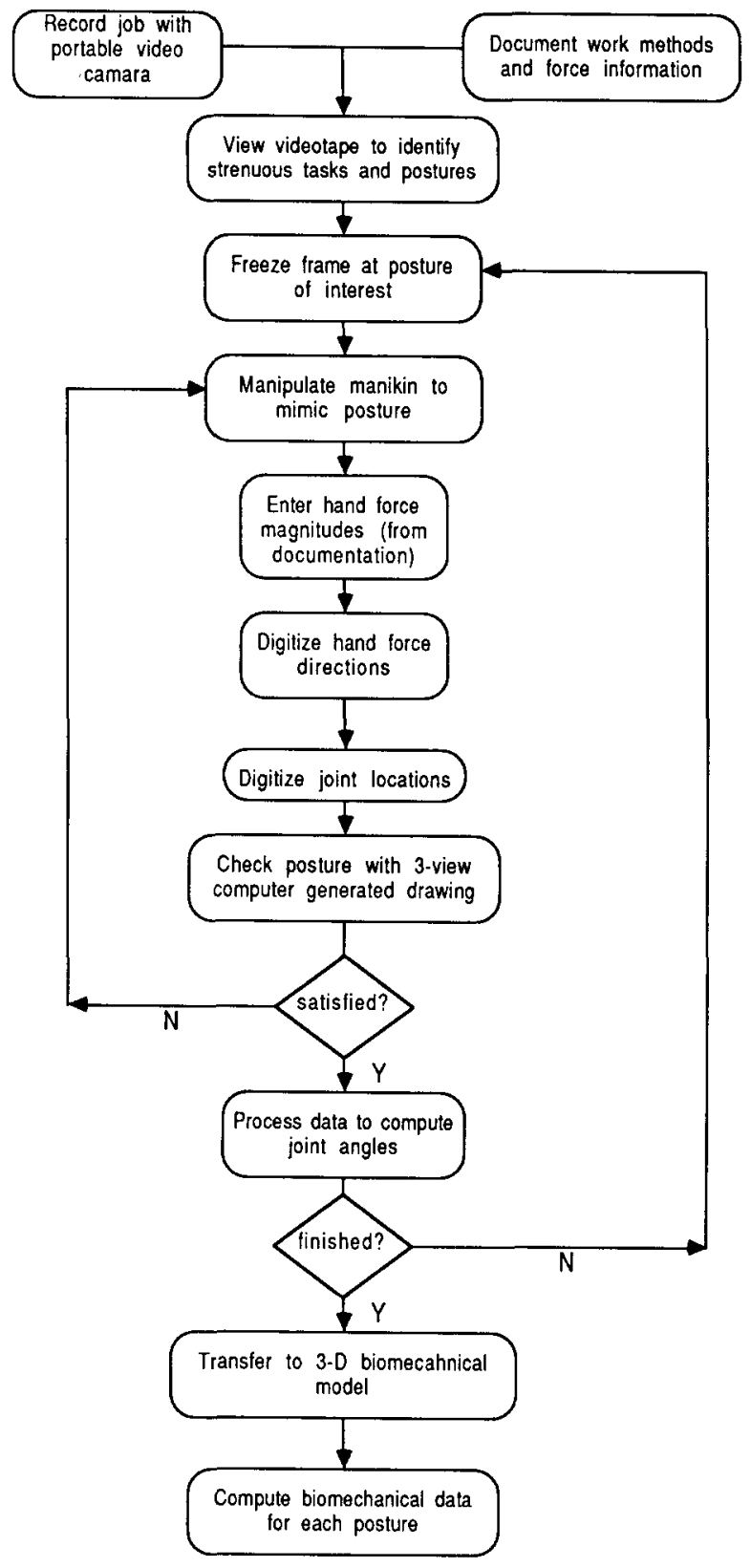

Fig. 3. Flowchart outlining the data collection procedure.

work activities may not follow a cyclical pattern. In these situations, it is necessary to produce videorecordings of all work sequences that involve physical effort.) In addition to making the tape, it is necessary to develop a brief description of work methods, equipment, and tools, and to measure the magnitude and direction of forces exerted by 
the operator during materials handling tasks.

To perform the analysis, the tape is played back at a workstation equipped with a personal computer, "space tablet" digitizer, and manikin. The analyst scans the tape, looking for tasks that involve significant physical exertions, such as lifting, carrying, pushing, or pulling. (The force measurements taken at the worksite assist the analyst in identifying these tasks.) When a task is identified, the tape is stopped to freeze the frame on the video monitor. (If desired, the analyst can take a photograph of the selected frame to document the posture selected for analysis.) The analyst then manipulates the manikin to simulate the posture of the worker on the videoscreen. When satisfied that the manikin is in the proper configuration, the location of the manikin's hands and major articulations (e.g. ankles, knees, hips, L5/S1, shoulders, and elbows) are entered using the space tablet pointer and digitized. The computer also queries the analyst to enter the direction and magnitude of the force exerted while in the posture.

The digiiized joint coordinates are then processed with the "POSTURE-3D" software package. This program generates the following outputs:

(a) A graphical representation of the encoded posture (top, side, and front views) is presented on the computer monitor. This allows the analyst to check the digitized posture against the posture seen on the videotape. If the analyst is not satisfied with the displayed posture, he uses the space tablet to re-enter the location of any incorrectly positioned joints. No further processing of the raw data occurs until the analyst is satisfied that all joints have been correctly entered and digitized.

(b) The digitized data are processed using a scaling subprogram to compute the Cartesian coordinates of the joint centres of a 50th percentile male in the depicted posture. (If desired, joint centres of other anthropometric standards could be computed simply by changing the scaling factors.) These coordinates are stored on diskette (University of Michigan, 1985).

(c) Vector algebra is used to convert the coordinates of the joint centres into the body angles required by the three dimensional strength prediction model (Garg and Chaffin, 1975). These angles are stored on diskette along with the magnitude and direction of the external force acting on the body.

(d) Joint angles and the external force vectors are transferred to a minicomputer (Hewlitt Packard 1000) which is the host system for the three dimensional biomechanical model. This model uses the body angles and force data to evaluate the strength demands of the selected task and posture, and to compute stresses acting at the L5/S1 disk.

The remainder of this paper presents and discusses an experiment performed to evaluate the nature and significance of measurement errors when using the new system to determine the body angles required for the three-dimensional strength prediction model.

\section{THE EVALUATION EXPERIMENT}

The objective of this experiment was to quantify and evaluate the variability of the body angle measurements obtained when using the new system. A $10 \times 5 \times 3$ full factorial analysis of variance was performed to evaluate the contributions to measurement variance due to the posture selected for analysis (ten levels), subject effects (five levels) and pure error (intra-subject differences on three repeated measures).

\section{Independent variables}

\section{Posture}

To evaluate the effectiveness of the system in distinguishing differences in joint angles associated with changes in gross body posture, working posture was selected as an independent variable. The test postures used in the experiment were selected from an extensive library of videotapes depicting work activities in an automobile assembly plant. Ten postures were selected to be representative of typical work activities. Transparencies (i.e., $2 \times 2$ slides) were made of these postures by photographing the appropriate "freeze frames" from the videotapes. The ten test postures, illustrated in Fig. 4 with tracings of the transparencies, included lifting activities (stooped, upright, and one-handed), carrying (one and two objects), pulling (above and below shoulder height), power tool operation (above and below shoulder height), and equipment operation. 

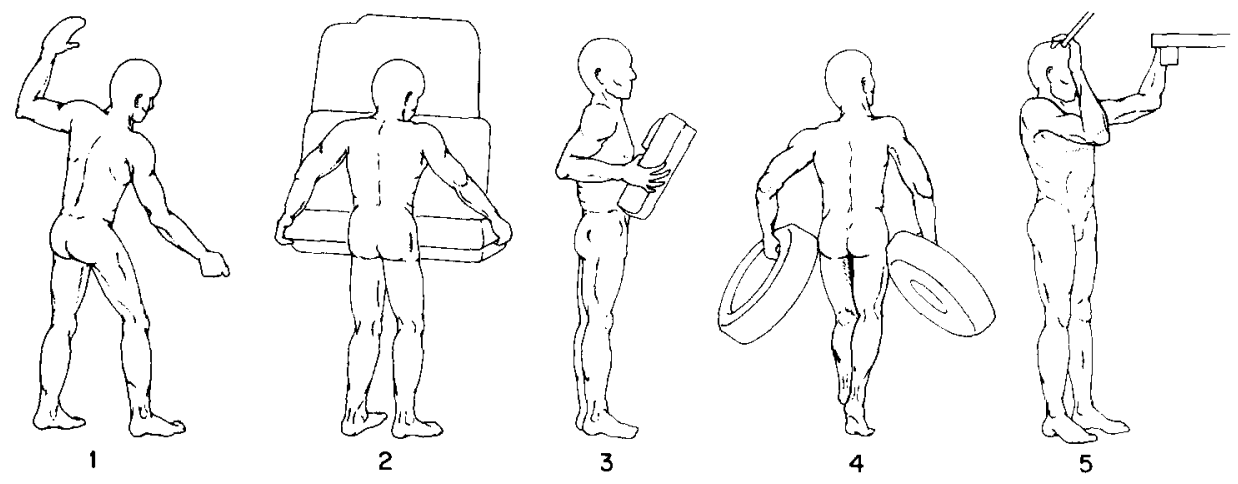

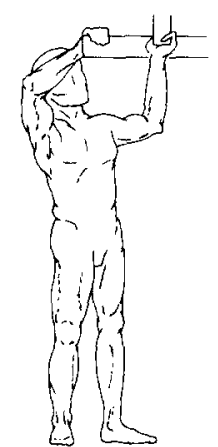

6
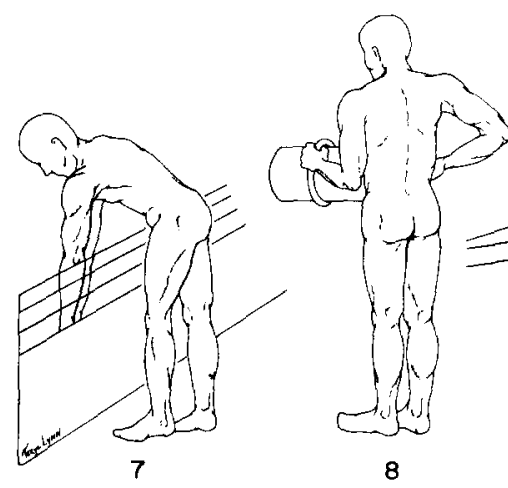

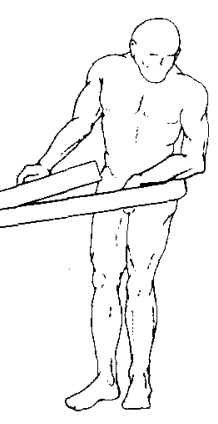

9

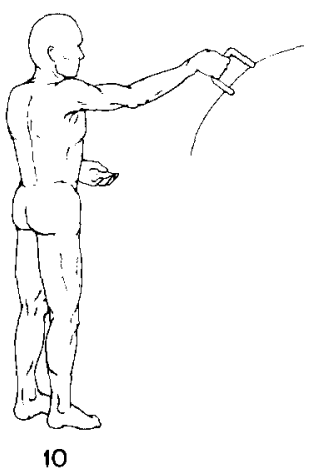

Fig. 4. Artist's sketches of the ten experimental postures.

\section{Analyst}

To evaluate inter-analyst variability, five subjects (three males, two females) were selected to participate in the experiment. All subjects were college students with some knowledge of ergonomics, biomechanics, and job evaluation. None of the students had any experience in using the digitizing system prior to the experiment. All subjects were volunteers and received compensation for participation in the experiment.

\section{Pure error}

To evaluate the pure error associated with the measurement system, every subject digitized each of the ten postures on three occasions.

\section{Dependent variables}

The dependent variables were the 15 angles required to define body posture at the elbows, shoulders, trunk, knees, and ankles. For an illus- tration and description of these angles, refer to Fig. $1 b$ and Table 1. All 15 angles were determined for each experimental condition.

\section{Procedures}

Each subject participated in six experimental sessions over a period of approximately three weeks. In most instances, sessions were limited to one per day per subject. The typical inter-session interval was two or three days, depending upon subject and equipment availability.

Each subject received individualized training during the first session. As part of this training, the experimentor:

(a) provided a brief explanation of the goals of the experiment.

(b) demonstrated manipulation of the three-dimensional manikin into a configuration which mimiced the posture of a worker in a projected transparency. 
(c) demonstrated operation of the computer, software, and space tablet.

(d) demonstrated operation of the transparency projector.

Although subjects were encouraged to develop their own digitizing procedures, the experimentor answered questions and provided suggestions with respect to manikin manipulations and the configuration of the space tablet arm. The experimentor did not, however, provide any feedback with respect to the subjects' abilities to mimic postures on the manikin.

Following training, each subject was allowed to practice using the system until comfortable with the procedures. At this point, the subject was told to proceed with the first trial, a practice session. At the end of the practice session the experimentor returned to the data collection room to answer questions and to schedule subsequent sessions.

Joint coordinate data collected during Sessions 2-6 were saved on diskette for further processing on the minicomputer to compute joint angles. Data from sessions 2 and 3 were not used in the final analyses, however, due to expected learning artifacts on early trials. Joint angle results from sessions 4-6 were analyzed using the BMDP2V and BMDP8V Analysis of Variance Programs (Dixon, 1985) on the University of Michigan Amdahl 5860 computer.

\section{RESULTS}

The ten work postures used for the experimental trials were "freeze frames" taken from videotapes of automobile assembly jobs. Because direct measurements of body angles were not taken at the time of videotaping, the true values of the 15 angles were not known. Instead, it was necessary to establish a best estimate of these angles using the responses of the five subjects. To do this, the mean value of the 15 responses (5 subjects $\times 3$ trials) was computed for each joint angle in each of the ten work postures. These mean angles are presented in Table 2 for each posture along with a grand average for all postures.

The entries in Table 2 for the shoulders (vertical, horizontal, and humeral rotation angle) and elbows (included angle) were similar for the left and right sides of the body in experimental postures $2,3,4$, and 7 . These results indicate a symmetric posture in the sagittal plane. Examination of the sketches in Fig. 4 confirms that postures $2,3,4$, and 7 were symmetric in the sagittal plane. Posture 6 was nearly symmetrical in the sagittal plane with the exception of more pronounced medial humeral rotation at the left shoulder relative to the right shoulder.

The right and left side angles in Table 2 for the knees and ankles were similar in all ten postures,

TABLE 2

Mean values of the upper extremity angles ( 5 subjects $\times 3$ trials) for the ten experimental postures

\begin{tabular}{|c|c|c|c|c|c|c|c|c|c|c|c|}
\hline & \multicolumn{11}{|c|}{ Test posture } \\
\hline & 1 & 2 & 3 & 4 & 5 & 6 & 7 & 8 & 9 & 10 & Average \\
\hline Rt elbow & 157 & 86 & 67 & 80 & 81 & 96 & 163 & 49 & 110 & 152 & 104 \\
\hline Lt elbow & 106 & 86 & 67 & 95 & 129 & 97 & 161 & 91 & 110 & 98 & 104 \\
\hline Rt shoul vert & 49 & 58 & 53 & 54 & 94 & 113 & 63 & 51 & 27 & 87 & 65 \\
\hline Lt shoul vert & 91 & 58 & 53 & 53 & 95 & 89 & 63 & 25 & 25 & 14 & 56 \\
\hline Rt shoul horiz & 30 & 4 & -39 & -16 & 85 & 63 & 77 & -75 & 35 & 80 & 24 \\
\hline Lt shoul horiz & 20 & 4 & -39 & -12 & 80 & 68 & 76 & 18 & 12 & -24 & 20 \\
\hline Rt shoul hum & 87 & 105 & 119 & 140 & 17 & 52 & 77 & 141 & 104 & 74 & 92 \\
\hline Lt shoul hum & 47 & 105 & 119 & 139 & 48 & 17 & 80 & 105 & 102 & 115 & 88 \\
\hline Trunk flex & 75 & 67 & 85 & 78 & 86 & 92 & 33 & 83 & 94 & 93 & 79 \\
\hline Trunk rot & -2 & -1 & 0 & 0 & -5 & -3 & -1 & 5 & 18 & 0 & 1 \\
\hline Trunk lat & 13 & -3 & 0 & 0 & 1 & 3 & 1 & -1 & -3 & 1 & 1 \\
\hline Rt knee & 150 & 156 & 159 & 158 & 161 & 158 & 140 & 156 & 156 & 161 & 155 \\
\hline Lt knee & 153 & 156 & 159 & 146 & 155 & 155 & 140 & 142 & 151 & 158 & 152 \\
\hline Rt ankle & 74 & 74 & 74 & 65 & 70 & 71 & 66 & 69 & 74 & 76 & 71 \\
\hline Lt ankle & 65 & 74 & 74 & 69 & 68 & 69 & 71 & 66 & 70 & 73 & 70 \\
\hline
\end{tabular}


indicative of sagittal symmetry in the joints and limbs of the lower extremity. This result was confirmed by the sketches of Fig. 4. Note the relatively narrow range of knee and ankle angles across the ten experimental postures. These results were consistent with the fact that all of the selected postures depicted standing operations with little flexion of the knees. See Fig. 4.

Analyses of variance were performed on the repeated measures design to evaluate the significance of the independent variables: subject and posture. The following model was used:

$Y_{i j k l}=\mu_{i . .}+S_{i j}+P_{i k}+\epsilon_{i j k l}$

where: $Y_{i j k l}$ is the measured angle at joint $i$ for subject $j$ on Posture $k$ for Trial $l ; \mu_{i} .$. is an overall constant for joint $i ; S_{i j}$ is the main effect of subject $j$ for joint $i ; P_{i k}$ is the main effect of posture $k$ for joint $i$; $\epsilon_{i j k l}$ is the measurement error (trial effect) associated with trial $l$ of subject $j$ and posture $k$ on joint $i ; i=1,2,3, \ldots 15 ; j=$ $1,2,3, \ldots 5 ; k=1,2,3, \ldots 10 ;$ and $l=1,2,3$. The BMDP2V Analysis of Variance Program (Dixon, 1985) was used to determine the significance of the independent variables at each of the 15 joints. The results of these analyses (simple significance levels with no corrections for multiple comparisons) are presented in Table 3.

Considering the results for the elbow and shoulder angles, posture was consistently found to be a highly significant factor $(p<0.0001)$, while subject in general was not significant. This finding was consistent with the expectations of a good measuring system; i.e., different postures produced different angles while different subjects generated similar angles.

A different pattern emerged in the results for the knees and ankles. For these lower body joints, subject was consistently a highly significant factor ( $p<0.0001$ at all four joints of interest). Posture was a significant factor for the knee angles $(p<$ 0.01 ), but not for the ankle angles. These results may be attributed to the fact that all of the experimental postures depicted standing workers thus narrowing the range of variance in the ankle angles. (See Fig. 4 and Table 2.) For additional explanations of these findings, refer to the Discussion section below.

The results at the trunk revealed yet another pattern. Subject and posture were significantly
TABLE 3

Significance levels of posture and subject for 15 joint angles

\begin{tabular}{llc}
\hline $\begin{array}{l}\text { Body joint } \\
\text { angle }\end{array}$ & \multicolumn{2}{l}{ Significance } \\
\cline { 2 - 3 } & Posture & Subject \\
\hline Rt elbow & $<0.0001$ & 0.03 \\
Lt elbow & $<0.0001$ & NS \\
Rt shoul vert & $<0.0001$ & NS \\
Lt shoul vert & $<0.0001$ & NS \\
Rt shoul horiz & $<0.0001$ & 0.04 \\
Lt shoul horiz & $<0.0001$ & NS \\
Rt shoul hum & $<0.0001$ & NS \\
Lt shoul hum & $<0.0001$ & NS \\
Trunk flex & $<0.0001$ & 0.01 \\
Trunk rot & NS & NS \\
Trunk lat & NS & NS \\
Rt knee & $<0.01$ & $<0.0001$ \\
Lt knee & $<0.01$ & $<0.0001$ \\
Rt ankle & NS & $<0.0001$ \\
Lt ankle & NS & $<0.0001$ \\
\hline
\end{tabular}

related to the measured trunk flexion angle; while neither of the independent variables was related to trunk rotation or lateral bending. This finding may have resulted from the fact that all of the experimental postures selected for the study required little or no deviation from neutral in the rotation and lateral-bending directions. (See Fig. 4 and Table 2.) These findings will be discussed in greater detail below.

To evaluate the relative contribution of the independent variables and pure measurement error to the total variance of the measured joint angles, it was necessary to partition the variance at each joint into its principal components:

$S_{i}^{2}=S_{S i}^{2}+S_{P i}^{2}+S_{S P i}^{2}+S_{E i}^{2}$

where: $S_{i}^{2}$ is the total measured variance at joint $i ; S_{S i}^{2}$ is the variance at joint $i$ due to subject effects; $S_{P i}^{2}$ is the variance at joint $i$ due to posture effects; $S_{S P i}^{2}$ is the variance at joint $i$ due to interaction (subject $\times$ posture) effects; $S_{E i}^{2}$ is the variance at joint $i$ due to pure (test-retest) error within a subject $\times$ posture treatment; and $i=$ $1,2,3, \ldots 15$. The BMDP8V Analysis of Variance Program (Dixon, 1985) was used to compute the components of variance described above. Simple coefficients of determination $\left(R^{2}\right)$ for subject effects $\left(S_{S i}^{2} / S_{i}^{2}\right)$, posture effects $\left(S_{P i}^{2} / S_{i}^{2}\right)$, and interaction effects $\left(S_{S P i}^{2} / S_{i}^{2}\right)$ were then computed using the ratios of the appropriate variance components. 
The results of these analyses are presented in Table 4 for the 15 body angles. Standard deviations of pure error (i.e., test-retest variability across the three trials for a given subject and posture) are also presented in the far right column of Table 4.

Looking first at the elbow angle, the standard deviations of pure error were similar for the right and left sides (14.2 degrees vs. 14.1 degrees). Pure error accounted for 10 percent of the variance on the right side and 19 percent of the variance on the left side. As would be expected from the significance levels presented in Table 3 , a large fraction of variance was explained by posture while a relatively small fraction of variance was explained by subject.

The results for the shoulder angles were less consistent. The standard deviations of pure error for the shoulder vertical angle were relatively small, 9.8 degrees for the right side and 10.1 degrees for the left side. Furthermore, a large fraction of the variance was explained by posture. Standard deviations of pure error were quite large for the shoulder horizontal angle, 31.1 degrees for the right side and 50.9 degrees for the left side. Pure error accounted for a large fraction of the variance, particularly on the left side. Finally, standard deviations of pure error and the contribution of pure error to total variance were relatively large for the humeral rotation angle on both sides of the body. Refer to the Discussion section below for an explanation of the relatively large pure error components associated with shoulder horizontal and humeral rotation angles.

Standard deviations of pure error for the three trunk angles (flexion, rotation, and lateral bending) were relatively consistent and relatively small. However, pure error accounted for a large fraction of total variance for the rotation and lateral bending angles. These apparently contradictory findings resulted from the selection of ten experimental postures where the work tasks involved virtually no major changes in trunk rotation or bending. With the exception of postures 8 and 9, none of the test postures depicted tasks involving trunk rotation; and with the exception of posture 1, none of the test postures depicted lateral bending. Furthermore, postures 1, 8, and 9 depicted only small deviations from a neutral posture. Due to the lack of systematic variance in trunk rotation and lateral bending, it was not surprising that posture accounted for only a small fraction of total variance. See Fig. 4 and Table 2. When the trunk posture varied substantially from neutral, the task required trunk flexion (e.g., posture 7). The results for trunk flexion show that a large fraction of total variance was explained by posture, with subject and pure error accounting for relatively small fractions of total variance.

Results for the knees and ankles were similar to the results for the trunk. The standard deviation

TABLE 4

Coefficients of determination $\left(R^{2}\right)$ for the independent variables and their first order interactions for the 15 angles

\begin{tabular}{|c|c|c|c|c|c|}
\hline \multirow{2}{*}{$\begin{array}{l}\text { Body joint } \\
\text { angle }\end{array}$} & \multicolumn{4}{|c|}{ Proportion of variance explained by: } & \multirow{2}{*}{$\begin{array}{l}\text { Std dev } \\
\text { pure error } \\
\text { (in degrees) }\end{array}$} \\
\hline & Subject & Posture & Subject posture & Pure error & \\
\hline Rt elbow & 0.03 & 0.81 & 0.06 & 0.10 & 14.2 \\
\hline Lt elbow & 0.05 & 0.59 & 0.17 & 0.19 & 14.1 \\
\hline Rt shoul vert & 0.01 & 0.80 & 0.07 & 0.12 & 9.8 \\
\hline Lt shoul vert & 0.01 & 0.85 & 0.03 & 0.11 & 10.1 \\
\hline Rt shoul horiz & 0.03 & 0.67 & 0.08 & 0.22 & 31.1 \\
\hline Lt shoul horiz & 0.04 & 0.28 & 0.16 & 0.52 & 50.9 \\
\hline Rt hum rot & 0.01 & 0.70 & 0.09 & 0.19 & 19.9 \\
\hline Lt hum rot & 0.01 & 0.59 & 0.10 & 0.30 & 26.6 \\
\hline Trunk inc & 0.05 & 0.69 & 0.06 & 0.20 & 9.5 \\
\hline Trunk rot & 0.03 & 0.08 & 0.33 & 0.56 & 11.2 \\
\hline Trunk lat & 0.04 & 0.05 & 0.06 & 0.85 & 12.4 \\
\hline Rt knee & 0.40 & 0.11 & 0.10 & 0.39 & 9.8 \\
\hline Lt knee & 0.32 & 0.15 & 0.07 & 0.46 & 10.1 \\
\hline Rt ankle & 0.48 & 0.04 & 0.07 & 0.41 & 7.9 \\
\hline Lt ankle & 0.33 & 0.03 & 0.04 & 0.60 & 8.1 \\
\hline
\end{tabular}


of pure error was small in all cases, but the contribution of pure error to total variance was relatively large. Furthermore, the contribution of posture to total variance was relatively small. This resulted from the lack of systematic variance in lower extremity joint angles in the ten experimental postures. See Fig. 4 and Table 2.

\section{DISCUSSION}

This study was a "first attempt" to develop and evaluate a computer-aided system for measuring three dimensional body angles from a two-dimensional video display. As a result of this study, insight was gained into sources of error when using the system. In general, the magnitude of pure error was reasonably consistent and small. With the exception of the shoulder horizontal and humeral rotation angles, the standard deviation for pure error (see Table 4) ranged between 7.9 and 14.2 degrees.

\section{Error in shoulder horizontal and humeral rotation angles}

The large errors in the shoulder horizontal angles can be attributed to the method used to define these angles rather than any inherent defect in the measurement system and/or method. The shoulder horizontal angle was measured in the transverse plane, and defined as the angle between the intersection of the frontal and transverse planes and the projection of the shoulder-to-elbow vector onto the transverse plane (Garg and Chaffin., 1975). This angle was defined to be positive if the elbow was positioned anterior to the frontal plane (i.e., if the shoulder was flexed from the anatomical position) and negative if the elbow was positioned posterior to the frontal plane (i.e., if the shoulder was extended). Using this definition, the sign of the shoulder horizontal angle instantly changed from positive to negative (or vice versa) when the elbow passed through the frontal plane. Assuming that the shoulder abduction angle was held constant during this movement, the magnitude of the angle would not change.

The most extreme effect of this definition on the measured angle occurs when the shoulder abduction angle is held at zero degrees. Under this condition, shoulder flexion of any magnitude results in a shoulder horizontal angle of $+90 \mathrm{de}-$ grees while shoulder extension of any magnitude results in a shoulder horizontal angle of -90 degrees. Even when the shoulder is mildly abducted, minor changes in the shoulder flexion/ extension angle will cause tremendous changes in the shoulder horizontal angle if the elbow passes through the frontal plane.

Many of the postures used in the experimental trials had the elbow positioned near the frontal plane. (See postures 2, 3, 4, 8, 9, and 10 in Fig. 4.) If, when manipulating the manikin to describe one of these postures, the elbow was not consistently positioned either anterior or posterior to the frontal plane on all three trials, pure error contribution to the variance of the shoulder horizontal angle was tremendously inflated due to changes in the sign of the measured angle.

The humeral rotation angle at each shoulder was determined by the relative positions of the shoulder, elbow, and hand. An algorithm using the shoulder vertical angle, shoulder horizontal angle, and the elbow-to-hand vector as predictor variables was used to determine this angle (Garg, 1973). Therefore, any pure error obtained in determining the shoulder horizontal angle was translated into pure error in the calculation of the humeral rotation angle.

\section{Measurement error}

There were several sources of error in the measurement system for all 15 angles. These were primarily attributed to: (a) the manikin/space tablet digitizing hardware and, (b) problems in interpreting three-dimensional information from a two dimensional display.

The principal problem with the manikin was that it utilized solid ball-and-socket joints at the articulations of interest. The diameter of these joints ranged from approximately $0.8 \mathrm{~cm}$ at the ankles to $2.7 \mathrm{~cm}$ at the lower back. Thus, the centre of rotation for each joint was located at the centre of a solid sphere. Because the space tablet pointer could not penetrate the sphere, the digitized joint centres were displaced from the true joint centres by a distance equal to the radius of the sphere. Because it was also impossible for subjects to consistently touch the same point on 
the sphere's surface from trial to trial, pure error was inflated.

The solid sphere design for joints also caused systematic modelling errors because the measured centre of joint rotation (located on the surface of the sphere) was not the true centre of joint rotation. The most obvious example of this type of error occurred at the ankles. Most of the test postures depicted standing workers where the ankle angle (i.e., the included angle between the foot and lower leg) was about 90 degrees. In digitizing the position of the ankle and knee, however, the subjects pointed the space tablet to the posterior surface of the ankle joint and the anterior surface of the knee joint. This practice produced an inclined orientation of the lower leg and reduced the measured ankle angle to about 70 degrees for most of the test postures. (See Table 2.) Subjects reported the solid sphere problem to be bothersome, particularly when digitizing the upper extremity angles.

A less serious problem with the manikin was the mechanical operation of the joints. In general, joints moved smoothly and manipulation of the manikin into the desired posture was easily accomplished. After prolonged use of the manikin, however, a few of the joints became "sticky" while others became "loose". As a result of this condition, it was sometimes difficult to manipulate the manikin into a desired posture and/or the manikin would not hold the desired posture long enough to digitize all joints.

As a result of these problems it is suggested that a new manikin be developed for future applications of this system. This manikin should utilize an integral goniometer at the true centre of rotation for each joint to measure and record joint angles and the orientation of adjacent limbs. This change would eliminate the bias and pure error problems associated with using the space tablet. The manikin should also use an improved mechanical design for the joints to eliminate problems with sticking or slippage.

Subjects reported difficulty in using the information provided by a two-dimensional display (i.e., a projected frame of a videotape) to generate a three-dimensional posture on the manikin. This was particularly true for joints with multiple motion axes, such as the trunk and shoulders. For example, test posture number 1 (see Fig. 4) involved simultaneous trunk flexion and lateral bending. It was unclear, however, how to resolve the two-dimensional picture into the three components of trunk motion. Similar problems were reported in an earlier study where subjects had difficulty in distinguishing trunk flexion from trunk bending (Keyserling, 1986).

Two approaches are suggested for resolving this problem. The first approach requires multiple cameras and a video mixer. The resulting videotape contains synchronized views of the job as recorded from two or more camera angles. By manipulating the manikin to simultaneously mimic multiple views, the three-dimensional fidelity of the simulated posture should be enhanced. The second approach is to allow the analyst to observe the job being performed in the workplace, preferably at the time that the videotape is made. Having once viewed the job in a true three-dimensional setting, an analyst should be better able to translate the two-dimensional video display into a three-dimensional manikin posture.

The perceptual aspect of interpreting posture was further complicated by the wearing of loosefitting coveralls and other types of protective clothing when performing the tasks depicted in the ten experimental postures. Subjects were instructed to "look through" the workers' clothing in order to estimate joint positions prior to manipulating the the manikin. According to informal reports from the subjects, this was not always easy to do. (Note: The sketches in Fig. 4 were an artist's conception of posture with clothing removed. In the original photographs, joint positions were covered.) Uncertainty due to clothing effects contributed to the variance of the measured angles in this experiment.

\section{Time requirements to use the system}

Following the three practice sessions, subjects were able to manipulate the manikin to mimic a posture, digitize joint locations with the space tablet, and run the required software programs in approximately three minutes per posture. When using the system in conjunction with the three-dimensional biomechanical strength model, additional time was needed to review the videotape to identify strenuous tasks. The amount of time required for a complete biomechanical analysis 
depended on the duration of the job and the number of strength demanding tasks. In a study of over 400 short-cycle (approximately 70 seconds) jobs in an automobile assembly plant, approximately 15 minutes were required per job.

\section{SUMMARY}

A new system for measuring three-dimensional postural angles was developed and tested on a limited basis. This system used a videotape to record posture in the workplace and a computeraided digitization system to measure the angles of 15 joints on playback. Approximately three minutes were required to use the system to describe a single posture.

Because this system works without in-plant calibration procedures and without attaching any instrumentation to the subject being studied, it can be used in the work environment with minimal interference to work activities and productivity. As such, it provides an efficient method for estimating work posture in situations where a high level of precision is not required.

\section{ACKNOWLEDGEMENTS}

The authors wish to thank Mr. James Foulke and Mr. Charles Woolley for their efforts in developing the hardware used in this study and Dr. Charles Anderson, Mr. Jeff Miller, Ms. Nancy Winfree, and Ms. Paula McEachern for their efforts in software development and data analyses. This research was supported in part through a research contract with the Ford Motor Company.

\section{REFERENCES}

Armstrong, T.J., Foulke, J.A., Joseph, B.A., and Goldstein, S.A., 1982. Investigation of cumulative trauma disorders in a poultry processing plant. Amer. Industr. Hygiene Assoc. J., 43: $103-116$.
Ayoub, M.M., Dryden, R.D., McDaniel, J.W., Knipfer, R.E. and Aghazadeh, F., 1978. Modelling of lifting capacity as a function of operator and task variables. In: Safety in Manual Materials Handling. NIOSH Pub. No. 78-185. National Institute for Occupational Safety and Health, Cincinnati (USA).

Chaffin, D.B., 1969. A computerized biomechanical model: development and use in studying gross body actions. $J$. Biomech., 2: 429-441.

Chaffin, D.B. and Park, K.S., 1973. A longitudinal study of low back pain as associated with occupational lifting factors. Amer. Industr. Hygiene Assoc. J., 34: 513-525.

Chaffin, D.B., Herrin, G.D., Keyserling, W.M. and Foulke, J.A., 1977a Pre-Employment Strength Testing. NIOSH Pub. No. 77-163. National Institute for Occupational Safety and Health, Cincinnati (USA).

Chaffin, D.B., Herrin G.D., Keyserling, W.M. and Garg, A., $1977 \mathrm{~b}$ A method for evaluating the biomechanical stresses resulting from manual materials handling jobs. Amer. Industr. Hygiene Assoc. J., 38: 662-675.

Corlett, E.N., Madeley, S.J. and Manenica, I., 1979. Posture targetting: a technique for recording working postures. Ergonomics, 22: 357-366.

Dixon, W.J., 1985. BMDP Statistical Software-1985. University of California Press, Berkeley (USA).

Garg, A., 1973. The Development and Validation of a 3-Dimensional Hand Force Capability Model. Department of Industrial and Operations Engineering, The University of Michigan, Ann Arbor (USA).

Garg, A. and Chaffin, D.B., 1975. A biomechanical computerized simulation of human strength. AIIE Transactions, 7: $1-15$.

Karhu, O., Kansi, P. and Kuorinka, I., 1977. Correcting working posture in industry: a practical method for analysis. Applied Ergonomics, 8: 199-210.

Keyserling, W.M., 1986. Postural analysis of the trunk and shoulders in simulated real time. Ergonomics, 29: 569-583.

National Institute for Occupational Safety and Health (NIOSH), 1981. Work Practices Guide for Manual Lifting. Pub. No. 81-122, Cincinnati (USA).

Nordin, M., 1982. Methods for Studying Work Load with Special Reference to the Lumbar Spine. University of Goteborg: Department of Orthopaedic Surgery, Goteborg, Sweden.

Persson, J, and Kilbom, A., 1983. VIRA-En Enkel Videofilmteknik for Registrering och Analys a Arbetsstallningar och Rorelser. National Board of Occupational Safety and Health: Work Physiology Unit, Solna (Sweden) 1.

Snook, S.H., 1978. The design of manual handling tasks. Ergonomics, 21: 963-985.

University of Michigan: Center for Ergonomics, 1985. POSTURE-3D Software for Three Dimensional Joint Angles. Ann Arbor (USA). 